Summarische Datengrundlagen reichen hierfür nicht aus. Da diese Regelung technisch hoch kompliziert und sehr konfliktbeladen ist, hat der Gesetzgeber ausdrücklich das Schiedsamt als eine Ebene der Konfliktlösung vorgesehen. Sofern, wie von allen Parteien übereinstimmend vorgetragen wurde, ein solches Schiedsamt noch nicht besteht, kann nicht im Wege der Vorwegnahme die Abschlagszahlung seitens der Ag. gekürzt werden, sondern es muss zunächst der formale Weg der Installierung des Schiedsamtes mit der anschließenden Anrufung desselben beschritten werden. Zudem kann eine Bereinigung auch mangels Vorgaben des Bewertungsausschusses für rechtskonforme Bereinigungslösungen für die Regelleistungsvolumina derzeit noch nicht durchgeführt werden.

Da der Anordnungsanspruch bereits mangels Rechtsgrundlage für die Kürzung der Abschlagszahlung wegen der Nichteinhaltung des formalen Verfahrens zu bejahen ist, bedurfte es im Rahmen des Einstweiligen Rechtsschutzes keiner Erörterung des Einwandes, der Hausarztvertrag sei wegen seines Abschlusses mit einem nicht privilegierten Vertragspartner nichtig.

Es besteht für die Ast. auch ein Anordnungsgrund, da ihr nicht zugemutet werden kann, die Entscheidung in der Hauptsache abzuwarten. Anordnungsgrund ist bei der Regelungsanordnung die Notwendigkeit zur Abwendung wesentlicher Nachteile. Vermieden werden soll, dass der Ast. vor vollendete Tatsachen gestellt wird, bevor er wirksamen Rechtsschutz erlangen kann. Der Anordnungsgrund liegt vorliegend in der Gefahr der Inanspruchnahme der Ast. durch die Vertragsärzte, die gegen die Ast. einen Anspruch auf Auszahlung der Gesamtvergütung haben. Die Ast. hat glaubhaft dargelegt, dass sie zum 11.5.2009 die Abschlagszahlungen an die Vertragsärzte zu leisten hatte. Mit Einbehalt der 40 Millionen $€$ werden der Ast. Gesamtvergütungsanteile vorenthalten, die diese nach $₫ 5$ Abs. 1 der Abrechnungsbestimmungen der Ast. in voller Höhe an ihre Mitglieder zu erbringen hat. Zwar hat die Ast. die Mitteilung der Regelleistungsvolumina unter einen Vorbehalt gestellt, so dass diese RLVs auch nach der Bereinigung angepasst werden können und keine Gefahr besteht, dass die betroffenen Ärzte wegen bestandskräftiger RLV auf eine Ausschöpfung derselben auch nach Bereinigung der MGV bestehen könnten. Dies ändert aber nichts an dem derzeitigen grundsätzlichen Anspruch der betroffenen Ärzte gegen die Ast.

Das Argument der Ag., die Hausärzte würden bereits durch den Hausarztvertrag bedient und hätten daher keinen Anspruch mehr gegen die Gesamtvergütung, geht insofern ins Leere, als die Höhe der konkreten Ansprüche der am Vertrag teilnehmenden Hausärzte erst nach der Durchführung des Bereinigungsverfahrens der MGV sowie der Regelleistungsvolumina errechnet werden kann. Zum derzeitigen Zeitpunkt ohne die durchgeführten Bereinigungen haben die Hausärzte grundsätzlich einen Anspruch auf Auszahlung der zum jetzigen Zeitpunkt festgelegten Gesamtvergütung gegen die Ast. Zudem ist nicht nachvollziehbar, warum die Abschlagszahlung um 40 Millionen $€$ gekürzt wurde, der Hausarztvertrag aber nur mit einer Abschlagszahlung von 34 bzw. 35 Millionen $€$ bedient wurde. Die Berechnung der 40 Millionen $€$ wurde zwar in einem Erörterungstermin erläutert, doch ist diese Summe deutlich höher als die an die Hausärzte zu leistende Abschlagszahlung und mit dem Argument der Vermeidung von Doppelfinanzierung nicht erklärbar.

Auch der Einwand der Ag., es erfolge keine Aufrechnung, sondern ein Auftrag zur Neuberechnung der durch die Veränderung des Sicherstellungsauftrages neu zu bestimmenden Gesamtvergütung, ändert nichts an dieser Beurteilung. Die Höhe der Gesamtvergütung wird durch Vereinbarung festgesetzt. Zur Bereinigung ist ein bestimmtes formales Verfahren vorgesehen, so dass nicht der Abschluss von Selektivverträgen kraft Gesetzes die Gesamtvergütung mindert. Vielmehr entsteht durch den wirksamen Abschluss von Selektivverträgen ein Anspruch auf Bereinigung, der als solcher aber noch nicht die Gesamtvergütung mindert.

Zudem besteht Wiederholungsgefahr, da die nächste $\mathrm{Ab}$ schlagszahlung für Mai Anfang Juni fällig wird.

Durch die vorläufige Verpflichtung der Ag., die Abschlagszahlung zunächst zu leisten, ist auch keine Vorwegnahme der Hauptsache erfolgt, Wie bereits mehrfach ausgeführt, ist die konkrete Bereinigung der Gesamtvergütung durch das vorgeschriebene formale Verfahren - unter Umständen mit Einschaltung des Schiedsamtes - durchzuführen.

Der Anspruch auf Prozesszinsen in Höhe von 5\% ergibt sich aus $\$ 291 \mathrm{BGB}$, insofern wird auf das Urt. des BSG v. 28.9.2005 - B 6 KA 71/04 R - verwiesen. [...]

\title{
BUCHBESPRECHUNGEN
}

DOI: 10.1007/s00350-009-2434-6

\section{Recht im Gesundheitswesen. Textsammlung.}

Herausgegeben von Walter Thürk. Verlag Carl Heymanns, Köln 2008, Stand der Ordner 1 u. 3: 19. Dez. 2007, Ordner 2 6. Dez. 2007, ca. 3286 S., $€ 99,00$

Es scheint an der Zeit, das ebenso umfassende wie bisher stets aktuelle und zudem preiswerte Werk des Direktors des Instituts für medizinische und pharmazeutische Prüfungsfragen und Ministerialrats a.D. Walter Thürk nach der 127. Lieferung vom Dezember 2008 wieder einmal insgesamt zu würdigen und damit zugleich der Erwartung Ausdruck zu geben, die Sammlung möge ihre Fortsetzung erfahren und die 128. Lieferung bald erscheinen können. Die Hinweise zu jeder Lieferung - die grundlegende entsprach dem Stande vom 1.2.1970 - hat der Herausgeber im ersten der drei Loseblatt-Leinenordner an den Anfang gestellt: sie ergeben zusammen eine kurz gefasste Geschichte des sich immer weiter entfaltenden und immer

Prof. Dr. iur. Dr. h.c. Adolf Laufs,

Heidelberg, Deutschland weniger fasslichen Gesundheitsrechts. „Der Thürk“ umgreift und erschließt diese Fülle.

Das Inhaltsverzeichnis nach Sachgebieten bietet auf vierzehn Seiten einen Überblick über den auf bewährte Weise in sechs Hauptkapitel gegliederten Stoff, der mehr als dreitausend Druckseiten beansprucht: 1. Organisation des Gesundheitswesens (Verfassungsrecht, Allgemeine Organisation, Bundeseinrichtungen); 2. Heilberufe (Ärzte, Zahnärzte, Heilpraktiker, Psychotherapeuten, Gemeinsamkeiten wie PartGG); 3. Gesundheitsberufe (Hebammen, Krankenpfleger, Technische Assistenten, Physiotherapeuten und viele andere - dazu überaus reichhaltig die jüngste Lieferung); 4. Apotheken- und Arzneimittelwesen, Medizinprodukte (mit dem durch Gesetze und Verordnungen geregelten Recht der Arzneimittel und am Ende dem HWG); 5. Krankheitsvorsorge und -bekämpfung (Impfen, Strahlenschutz, Gesundheitsfürsorge mit u.a. ESchG, KRG, HiVHG, TPG, StZ); 6. Krankenhauswesen (Krankenhausentgelte und Krankenhausfinanzierung). Das zwanzigseitige alphabetische Inhaltsverzeichnis führt von „Abgrenzung“ bis „Zuständigkeit“. Als hilfreich erweisen sich auch die knappen Fußnoten des Herausgebers mit kurzen Notizen insbesondere zum Inkrafttreten der Rechtssätze und zu ihren Änderungen.

Der Rezensent wiederholt gern sein Urteil in MedR 2006, 474 und bekennt, dass ihm die Edition seit langem ein unentbehrliches Arbeits- und Auskunftsmittel ist. 